\title{
TT a magnum of Laurent-Perrier Champagne!
}

Rearrange the letters in the purple squares to find the secret word (7). Complete the answer form and send to the following address by 7 May 2010: Vital, BDJ Editorial, NPG, 4-6 Crinan Street, London N1 9XW.

The answers and name of the prize winner will be announced on 12 June 2010, when the summer

\begin{tabular}{|c|c|c|c|c|c|c|c|c|c|c|c|}
\hline S & $B$ & $S$ & & $T$ & $\mathrm{U}$ & $B$ & $\mathrm{~B}$ & 0 & & & issue of Vital is \\
\hline $\bar{Q}$ & $U$ & & & & & L & & $R$ & & $\mathrm{~J}$ & published. \\
\hline $\mathrm{U}$ & $\mathrm{D}$ & E & $R$ & G & & $\mathrm{A}$ & & $A$ & $R$ & 2 & Sarah \\
\hline 1 & $\mathrm{D}$ & & 0 & & & $\mathrm{~s}$ & & $\mathrm{~T}$ & & $E$ & Hodgson, a \\
\hline 0 & $\mathrm{Y}$ & A & $\mathrm{L}$ & $\underline{P}$ & & $\mathrm{~T}$ & $\mathrm{H}$ & O & S & & dental nurse \\
\hline$R$ & & & & & & & & $R$ & & & from Cranleigl \\
\hline $\mathrm{E}$ & $P$ & E & c & $T$ & & A & & $\bar{Y}$ & E & & \\
\hline $\mathrm{L}$ & $\mathrm{A}$ & & & $\underline{\underline{n}}$ & & $\mathrm{~N}$ & & & & כ & Secretwerd \\
\hline & $\mathrm{R}$ & $\mathrm{E}$ & A & $\mathrm{H}$ & & $\mathrm{s}$ & M & A & & L & secretword \\
\hline$s$ & $\Gamma_{-}$ & & $\mathrm{R}$ & & & $\mathrm{w}$ & & $\mathrm{L}$ & & I & (see left). The \\
\hline $\mathrm{T} / \mathrm{E}$ & $\mathrm{N}$ & $\mathrm{s}$ & $\mathrm{E}$ & $\underline{S}$ & $\mathrm{H}$ & $\mathrm{E}$ & $\mathrm{R}$ & I & & & secret word \\
\hline O & $\mathrm{E}$ & & $\mathrm{N}$ & 트 $>2+$ & & $\mathrm{R}$ & & $\underline{B}$ & & & was ENAMEL. \\
\hline \begin{tabular}{l|l}
$P$ & $O$ \\
\end{tabular} & $R$ & & $\mathrm{~A}$ & B $L$ & $E$ & & D & & & & \\
\hline
\end{tabular}

\section{Just for fun Wordsearch}

\begin{tabular}{|c|c|c|c|c|c|c|c|c|c|}
\hline O & H & E & D & N & A & L & L & O & H \\
\hline B & S & A & F & E & T & Y & R & E & O \\
\hline D & E & A & F & W & I & S & E & S & S \\
\hline H & T & L & Y & N & N & P & T & R & P \\
\hline E & I & A & A & T & H & L & H & E & I \\
\hline A & S & T & E & A & M & A & I & N & T \\
\hline L & B & S & I & D & L & Q & C & N & A \\
\hline T & E & O & D & C & I & U & S & I & L \\
\hline H & W & P & P & P & Z & E & N & W & S \\
\hline B & A & R & C & O & D & I & N & G & G \\
\hline
\end{tabular}

Taken from this issue of Vital...

$\begin{array}{lll}\text { DEAFWISE } & \text { WEBSITES } & \text { CPD } \\ \text { LIZ } & \text { HOSPITALS } & \text { OHE } \\ \text { LYNN } & \text { HOLLAND } & \text { WINNERS } \\ \text { DCP } & \text { HEALTH } & \text { PLAQUE } \\ \text { ETHICS } & \text { SAFETY } & \text { TEAM } \\ \text { BARCODING } & \text { POSTAL } & \end{array}$

When you have found all of the words in the above list, the remaining letters read from top to bottom will spell out a hidden word which is the subject of an article in this issue (9).

Winter issue hidden word: Inspiring

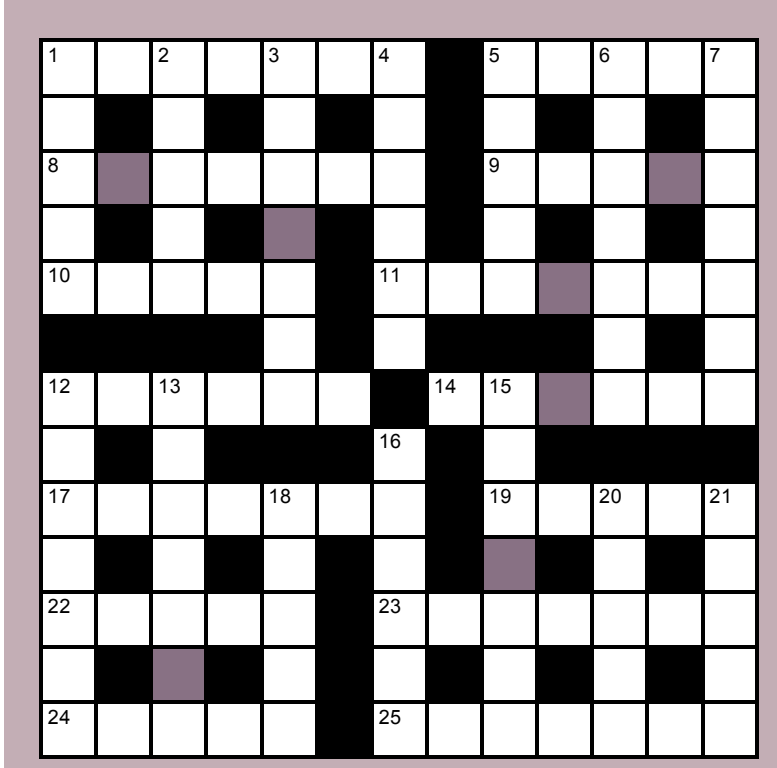

\section{Across}

1 Stashed away (7)

5 Allure (5)

8 Release air from (7)

9 Bury (5)

10 Acclaim (5)

11 Quieten (7)

12 Large flat food fish (6)

14 Pre-Christmas season (6)

17 Commander of a fleet (7)

19 Elephant tusk (5)

22 Thick short hand digit (5)

23 Out loud (7)

24 Underground burial chamber (5)

25 Perspired (7)
Down

1 Garden boundary (5)

2 Terrible (5)

3 Forceful (7)

4 Smartly turned out (6)

5 Court proceedings (5)

6 Afternoon theatrical performance (7)

7 Tease (7)

12 Credit card (colloq.) (7)

13 Weapons store (7)

15 Become smaller (7)

16 Forever (6)

18 Automaton (5)

20 Revolve around (5)

21 Give up (5)

AITLE
ADST NAME
EMSTAL CODE
JOB TITLE
SECRET WORD
If you prefer not to be contacted for purposes other than this competition, please
tick this box. We will not pass your details on to any third parties.

